$@$ ERSpublications

Radiation therapy remains an alternative treatment option for post-surgical lung cancer relapse http://ow.ly/GaM43006HDs

Dragan Subotic ${ }^{1}$, Paul Van Schil ${ }^{2}$ and Bogdan Grigoriu ${ }^{3}$

${ }^{1}$ Clinic of Thoracic Surgery, Clinical Center of Serbia, University of Belgrade Faculty of Medicine, Belgrade, Serbia.

${ }^{2}$ Dept of Thoracic and Vascular Surgery, University Hospital, Antwerp, Belgium. ${ }^{3}$ Dept of Pulmonology, University of

Medicine and Pharmacy, Iasi, Romania.

Correspondence: Dragan Subotic, Clinic of Thoracic Surgery, Clinical Center of Serbia, University of Belgrade Faculty of Medicine, Koste Todorovica 26, 11000 Belgrade, Serbia. E-mail: profsubotic@gmail.com

Received: April 292016 | Accepted: May 042016

Conflict of interest: None declared.

\title{
References
}

1 Subotic D, Van Schil P, Grigoriu B. Optimising treatment for post-operative lung cancer recurrence. Eur Respir J 2016; 47: 374-378.

2 Jeremic B, Shibamoto Y, Milicic B, et al. External beam radiation therapy alone for loco-regional recurrence of non-small-cell lung cancer after complete resection. Lung Cancer 1999; 23: 135-142.

3 Cai XW, Xu LY, Wang L, et al. Comparative survival in patients with postresection recurrent versus newly diagnosed nonsmall-cell lung cancer treated with radiotherapy. Int J Radiat Oncol Biol Phys 2010; 76: 1100-1105.

4 Takenaka T, Takenoyama M, Toyozawa R, et al. Concurrent chemoradiotherapy for patients with postoperative recurrence of surgically resected non-small-cell lung cancer. Clin Lung Cancer 2015; 16: 51-56.

5 Jeremic B, Bamberg M. External beam radiation therapy for bronchial stump recurrence of non-small-cell lung cancer after complete resection. Radiother Oncol 2002; 64: 251-257.

6 Van Schil PE, Van Meerbeeck J. Surgery or radiotherapy for early-stage lung cancer - a potential comparison bias. Lancet Oncol 2013; 14: e390.

7 Van Schil PE. Results of surgery for lung cancer compared with radiotherapy: do we speak the same language. J Thorac Oncol 2013; 8: 129-130.

Q

CrossMark

\section{Readmission in COPD patients: should we consider it a marker of quality of care or a marker of a more severe disease with a worse prognosis?}

\section{To the Editor:}

In the January edition of the European Respiratory Journal, we read with interest the paper by HARTL et al. [1], which evaluated, from the European COPD Audit, patients with acute exacerbation of chronic obstructive pulmonary disease (AECOPD) and the mortality risk during hospitalisation and in the post-discharge period of 90 days. Moreover, the authors evaluate the risk of readmission in a period of 90 days from discharge.

The readmission to hospital that occurs with COPD patients is a many-sided phenomenon, where the complexity of patients and the difficulties with the healthcare systems are often considered as causes [2]. The reasons of readmission may be respiratory and non-respiratory related [2]. Starting from the concept that in COPD patients, like for other chronic diseases with high costs related to discharge (congestive heart failure, acute myocardial infarction and pneumonia), the early readmissions (30-days from discharge) should be considered a marker of quality of care that patients receiving during hospitalisation [3], in USA by the Centres for Medicare and Medicaid Services financial penalties were applied for hospitals with high rates of readmissions [4]. Several interventions have been proposed [5], even if a single disease-specific approach is not recommended [2,5]; obviously, data about prediction of readmission might be extremely useful $[6,7]$.

Although the study by HARTL et al. [1] focuses on a period of 90 days from discharge and the causes of readmission are not exclusively respiratory related, our recently published long-term observational study [8] 
found similar results that we feel need some remarks. In a Spanish cohort of 378 AECOPD patients we found, in fact, that the readmission in a period of 30 days from discharge for a new AECOPD increase progressively the mortality risk in four periods of follow-up (30 days, 6 months, 1 year, and 3 years) [8]. Our finding supports the hypothesis that COPD patients with frequents events of AECOPD definite from the literature as "frequent exacerbation phenotype" [9] and reported from us as patients that need a readmission to hospital have a worse prognosis in comparison to patients without frequent and repeated events of AECOPD (no-readmitted patients); this aspect is furthermore true in a long-term follow-up.

Data on the higher prevalence of readmitted patients in the study by HARTL et al. [1] study in comparison to our study (35 versus 18\%, respectively) is clearly related to the larger period of observation from discharge used from the European COPD Audit (90 days versus 30 days). In the same context, the trend in the percentage of deaths among readmitted and no-readmitted appears to be totally in line to our data. HARTL et al. [1] in fact found higher death rates in readmitted patients in comparison to patients who were not readmitted (13.4 versus $2.3 \%$ ). This is similar to what our study showed at the 30 -day (5 versus $1 \%$ ), 6-month (27 versus 10\%), 1-year (37 versus 17\%), and 3-year (67 versus 43\%) follow-up periods, for readmitted patients and non-readmitted patients, respectively. In conclusion, COPD patients with a readmission as well at 30 days as at 90 days from discharge, have a worse prognosis.

Moreover, the risk associated with a 90-days post-discharge mortality, we note with interest in the logistic regression model of HARTL et al. [1] that the age, respiratory acidosis (mild or severe versus normal), the need of ventilator support, the Charlson index, and a previous admission are independent variables. Similarly we found in our univariate Cox regression model that the age ( $\geqslant 70$ years), the Charlson index $(\geqslant 3$ points), $\geqslant 2$ exacerbations requiring hospitalisation in a 1-year period prior to index hospitalisation and the reduction of $\mathrm{pH}$ level predict the probability of death at 1 year. However, these variables lost their significance in our multivariate model where significant independent predictors of mortality are the presence of a 30-day readmission, the partial arterial carbon dioxide pressure and the dyspnoea perception, both measured at admission. The difference in sample size for the two studies may explain the full tendency of results without a perfect correspondence of data in the multivariate analysis. Therefore, we may conclude that the severity of underling clinical condition of AECOPD patients may have an influence of prognosis.

Finally, we note the reported increased susceptibility of some COPD patients to have frequent AECOPD $[9,10]$, that a previous admission $(\geqslant 1)$ reported by HARTL et al. [1] is conceptually similar to our variable " $\geqslant 2$ previous exacerbations" in the model predicting readmission risk at 90 days and 30 days, respectively.

Based on this evidence, concerning the readmission as a phenomenon related to severity and then prognosis of COPD patients, a question that we should ask is: should we continue to think that in COPD applying financial penalties for hospital having high-rate readmission may solve the problem of readmission? Instead of penalties a better and close follow-up after discharge is strongly encouraged in the population at risk.

0

@ERSpublications

Readmission in COPD patients: a marker of quality of care or a more severe disease with a worse prognosis? http://ow.ly/ZwIpW

Ernesto Crisafulli ${ }^{1}$, Mónica Guerrero ${ }^{2}$, Alfredo Chetta $^{1}$ and Antoni Torres ${ }^{2}$

${ }^{1}$ Dept of Clinical and Experimental Medicine, Respiratory Disease and Lung Function Unit, University of Parma, Parma, Italy. ${ }^{2}$ Respiratory Dept, Clinic Institute of Respiratory (ICR), Hospital Clinic of Barcelona - Institut d'Investigacions Biomèdiques Agusti Pi i Sunyer (IDIBAPS) - CIBERES - University of Barcelona (UB), Barcelona, Spain.

Correspondence: Ernesto Crisafulli, Dept of Clinical and Experimental Medicine, Respiratory Disease and Lung Function Unit, University of Parma, Via Rasori 10, 43126 Parma, Italy. Email: ernesto.crisafulli@unipr.it

Received: Feb 282016 | Accepted: March 132016

Conflict of interest: None declared.

\section{References}

1 Hartl S, Lopez-Campos JL, Pozo-Rodriguez F, et al. Risk of death and readmission of hospital-admitted COPD exacerbations: European COPD Audit. Eur Respir J 2016; 47: 113-121.

2 Shah T, Churpek MM, Coca Perraillon M, et al. Understanding why patients with COPD get readmitted: a large national study to delineate the Medicare population for the readmissions penalty expansion. Chest 2015; 147: 1219-1226.

3 Department of Health and Human Services. Centers for Medicare and Medicaid Services. Medicare Program; Hospital Inpatient Prospective Payment Systems for Acute Care Hospitals and the Long-Term Care Hospital Prospective Payment System and Proposed Fiscal Year 2014 Rates; Quality Reporting Requirements for Specific Providers; Hospital Conditions of Participation. Federal Reg 2013; 78: 27823-27486. 
4 Feemster L, Au D. Penalizing hospitals for chronic obstructive pulmonary disease readmissions. Am J Respir Crit Care Med 2014; 189: 634-639.

5 Prieto-Centurion V, Markos MA, Ramey NI, et al. Interventions to reduce rehospitalizations after chronic obstructive pulmonary disease exacerbations. A systematic review. Ann Am Thorac Soc 2014; 11: 417-424.

6 Crisafulli E, Torres A, Huerta A, et al. C-reactive protein at discharge, diabetes mellitus and $\geqslant 1$ hospitalization during previous year predict early readmission in patients with acute exacerbation of chronic obstructive pulmonary disease. COPD 2015; 12: 306-314.

7 Crisafulli E, Ortega S, Torres A. Predictors of readmission in a period of 30 days or less in acute exacerbation of chronic obstructive pulmonary disease. Clin Pulm Med 2015; 22: 172-176.

8 Guerrero M, Crisafulli E, Liapikou A, et al. Rateadmission for acute exacerbation within 30 days of discharge is associated with a subsequent progressive increase in mortality risk in COPD patients: a long-term observational study. PLoS One 2016; 11: e0150737.

9 Wedzicha JA, Brill SE, Allinson JP, et al. Mechanisms and impact of the frequent exacerbator phenotype in chronic obstructive pulmonary disease. BMC Med 2013; 11: 181.

10 Hurst JR, Vestbo J, Anzueto A, et al. Susceptibility to exacerbation in chronic obstructive pulmonary disease. N Engl J Med 2010; 363: 1128-1138.

Eur Respir J 2016; 48: 279-281 | DOI: 10.1183/13993003.00426-2016 | Copyright @eES 2016

From the authors:

We thank Ernesto Crisafulli, Mónica Guerrero, Alfredo Chetta and Antoni Torres for their differentiated perspective on the topic of hospital readmission of chronic obstructive pulmonary disease (COPD).

We agree that hospital readmission is a complex surrogate parameter whereby the short period after dismission is related to a fragile health condition of the patient. The close correlation of readmission to increased mortality is another indicator of this vulnerable condition of the COPD patient, following acute exacerbation.

The pilot project of the European audit analysed only readmissions of the COPD patients due to respiratory reasons, in order to assess the risk related to the original hospital admitted exacerbation. This was also very well described in the data by HURST et al. [1], who demonstrated that the first exacerbation is followed by a second in nearly one-third of patients within 8 weeks. Moreover, we found a correlation of the readmission rate and the severity of respiratory failure during the first admission and in line with other publications, a consecutive increase in mortality after readmission [2-4]. GUERRERo et al. [5] reported a correlation with hypercapnia but not with the rate of ventilatory support, not even with admission to intensive care units. This is opposite to our study, which relates the risk of readmission to the severity of the exacerbation (respiratory acidosis, need of ventilation) and not only to disease severity. The need for ventilatory support is an expression of reduced ventilatory capacity in the acute situation and we know that muscle fatigue and overload in accordance with systemic corticosteroids have a potential for critical illness myopathy or neuropathy $[6,7]$. Therefore, in the short course after exacerbation ventilatory support was identified as an indicator of an instable condition and a risk factor for readmission.

Due to the observational character of the European COPD Audit study we are not able to predict the potential for the prevention of readmission or death by a single measure of care. However we observed a huge variation of care within each participating country and between countries according to the treatment of acute respiratory failure and the referral to rehabilitation after discharge. The analysis of the level of adherence to management recommendations of acute exacerbation of COPD showed a disappointing result of only $15 \%$ of accordance with suggested standard procedures [8]. These findings mirror the ignorance towards the challenge of acute exacerbation of COPD on admission as well as a passive attitude towards ongoing COPD management after discharge. The complexity of COPD exacerbation and of COPD patients overall should not result in reduced treatment approaches.

Therefore, we think that it is high time to accept hospital admitted acute COPD-exacerbation as a risk profile that needs a strict acute care programme and a secured follow-up management starting with post-acute rehabilitation including noninvasive ventilation and long-term oxygen therapy for according patients.

Complexity management is requested as a holistic approach in knowledge-management driven programmes, according to the combination of each single evidence-based intervention that may be suitable to the patients' risk profile as suggested by KirsCHNER et al. [9].

The assessment of quality of care is a powerful tool to monitor the changes in management and promote the discussion of care models. MCCARTHY et al. [10] showed a successful implementation of an improved COPD management at the emergency department after identification of weaknesses. The pilot of The European Audit intends to be a start for such activities. 